\title{
Accuracy of the hypospadias diagnoses and surgical treatment registrations in the Danish National Patient Register
}

This article was published in the following Dove Press journal:

Clinical Epidemiology

3 October 2017

Number of times this article has been viewed

\author{
Linn Håkonsen Arendt ${ }^{1,2, *}$ \\ Andreas Ernst ${ }^{1,3, *}$ \\ Morten Søndergaard \\ Lindhard ${ }^{2}$ \\ Anne Aggerholm Jønsson' \\ Tine Brink Henriksen ${ }^{2}$ \\ Jørn Olsen ${ }^{4}$ \\ Jorgen Thorup ${ }^{5}$ \\ L Henning Olsen ${ }^{3}$ \\ Cecilia Høst \\ Ramlau-Hansen' \\ *These authors contributed equally to \\ this work \\ 'Department of Public Health, Section \\ for Epidemiology, Aarhus University, \\ Aarhus, Denmark; ' Department of \\ Paediatrics and Adolescent Medicine, \\ Aarhus University Hospital, Aarhus, \\ Denmark; ${ }^{3}$ Department of Urology, \\ Section for Paediatric Urology, Aarhus \\ University Hospital, Aarhus, Denmark; \\ ${ }^{4}$ Department of Clinical Epidemiology, \\ Aarhus University Hospital, Aarhus, \\ Denmark; ${ }^{5}$ Department of Paediatric \\ Surgery, Rigshospitalet and Institute \\ of Clinical Medicine, University of \\ Copenhagen, Copenhagen, Denmark
}

Correspondence: Linn Håkonsen Arendt Department of Public Health, Section for Epidemiology, Aarhus University, Bartholins Allé 2, 8000 Aarhus C,

Denmark

Email lha@ph.au.dk
Purpose: The Danish National Health registers provide a valuable data source that offers unique opportunities for observational research, including studies on the congenital anomaly hypospadias. The accuracy of the diagnosis and surgical treatment registration of hypospadias in the Danish National Patient Register (DNPR) remains unknown.

Patients and methods: We randomly sampled 500 patients diagnosed with hypospadias in the DNPR from January 1, 1995 to December 31, 2012. Among these, 384 patients were also registered with surgical treatment for hypospadias. Medical records were collected and reviewed independently by two investigators. Any classification disagreements were resolved by consensus. Using the medical records as the gold standard, we estimated positive predictive values (PPVs) with 95\% confidence intervals (CIs) for the hypospadias diagnoses and surgical treatment registrations overall, as well as for the clinical subtypes.

Results: We were able to retrieve medical records for 463 (92.6\%) patients with hypospadias diagnoses and for $329(85.7 \%)$ patients registered with surgical treatment. Presence of hypospadias was confirmed in 450 of 463 patients, yielding an overall PPV (95\% CI) of 97.6\% (95.8\%-98.7\%). For subtypes of hypospadias, the PPVs ranged between $37.5 \%$ and $72.7 \%$. For surgical treatment of hypospadias, the overall PPV was 99.7\% (97.9\%-99.9\%).

Conclusion: The validity of the registration of hypospadias diagnoses as well as surgical treatment for hypospadias in the DNPR is overall very high. For the specific subtypes of hypospadias diagnoses codes and the specific surgical treatment codes, the PPVs are lower and cautious use is warranted. However, the DNPR remains a valuable tool for future observational research on hypospadias.

Keywords: data quality, epidemiology, hospital register diagnoses, ICD-10, positive predictive value

\section{Introduction}

The Nordic national health registers provide a highly valuable population-based data resource for observational research. For five decades, the Danish Civil Registration System has assigned a unique personal identification number to all Danish residents, ${ }^{1,2}$ enabling individual level linkage of data from registers, medical databases, and birth cohorts. The Danish National Patient Register (DNPR) holds information on all hospital contacts with complete nationwide coverage since $1978 .{ }^{3,4}$ It is among the most commonly used health-care register in Denmark and enables researchers to perform nationwide, population-based observational studies within various research fields. Research on rare conditions such as congenital abnormalities also benefits from these long-term follow-up data. 
Hypospadias is a congenital anomaly of the male genitalia with an estimated prevalence of $0.5 \%-0.8 \%$ in Denmark. ${ }^{5}$ It is recognizable by incomplete closure of the urethra leading to various degrees of displacement of the external urethral orifice along the ventral side of the penis, scrotum or perineum, often accompanied by abnormal foreskin and ventral penile shaft deviation. ${ }^{6}$ In recent years, there has been a large research focus on temporal trends, etiology, and risk factors for hypospadias as well as long-term consequences for the affected boys. ${ }^{7}$ Danish register-based research has contributed substantially to the current knowledge in this area.,8-14 Yet, many etiological aspects and research questions remain unanswered and register-based data will continuously serve as an important tool in answering these questions in the future.

Although the overall validity of the DNPR is considered high, not all diagnoses have been shown to be accurate. ${ }^{15}$ The accuracy of the hypospadias diagnosis and surgical treatment in the DNPR remains unknown.

\section{Material and methods}

\section{Setting and data collection}

In this nationwide, population-based validation study, we assessed the accuracy of the International Classification of Diseases (ICD-10) discharge diagnosis codes and surgical treatment codes for hypospadias in the DNPR between January 1, 1995 and December 31, 2012. The DNPR was established in $1977^{3,4}$ and provides complete nationwide information on all inpatient contacts to Danish hospitals since 1978 and on outpatient contacts since 1995. From 1977 to 1995, diseases were classified according to the ICD-8 and from 1995 and onward, the ICD-10 has been used.

All patients registered with a main inpatient or outpatient hospital discharge ICD-10 code of hypospadias (Q54; hypospadias glandis [Q540], hypospadias coronalis [Q540A], hypospadias corporis penis [Q541], hypospadias penoscrotalis [Q542], hypospadias perinealis [Q543], other specified hypospadias [Q548], and hypospadias without specifications [Q549]) between January 1, 1995 and December 31, 2012 were identified. Among all registered in the DNPR, the Danish Statens Serum Institut randomly selected a representative sample of 500 patients. From the DNPR, we obtained information on the unique civil registration number, date of admission, date of operation, date of discharge, hospital code, departmental code, diagnostic specialty, all main and secondary diagnoses codes for hypospadias and other congenital anomalies or genetic syndromes (ICD-10: Q00-Q99) as well as surgical treatment codes for hypospadias; operation for hypospadias (KGH60), glandular hypospadias (KGH60A), corporal hypospadias (KGH60B), penoscrotal hypospadias
(KGH60C), and perineal hypospadias (KGH60D) for the randomly selected study population of 500 patients. Thus, we studied the accuracy of registration of surgical treatment among those registered with a hypospadias diagnosis.

Medical records were collected from June 2015 to September 2016. Copies of the medical records were requested by mail directed to the department responsible for the first hypospadias registration, as well as to the hospital performing the surgical treatment for hypospadias. Reminders were mailed to the non-responding departments after approximately 3 months and, finally, the remaining departments were contacted by phone and/or personal visits.

\section{Medical record review}

From October 2016 to February 2017, two investigators independently reviewed each medical record, blinded to the diagnoses and surgical treatment codes registered in the DNPR. Data were extracted using scoring protocols developed in collaboration with experts within pediatric urology and then entered in the Research Electronic Data Capture (REDCap) software. ${ }^{16}$ The physicians' descriptions in the medical records served as the gold standard. When discrepancies related to the location of the external urethral orifice existed, the description of urethral orifice according to the surgical operation report was superior. If the patient did not receive surgical treatment or if the description was missing, the description from the outpatient clinic was used. In the ICD-10, the condition penis arcuatus (Q544) is registered as a subtype of hypospadias. However, as isolated penile shaft deviation without urethral meatus displacement mainly originates due to various reasons other than hypospadias, penis arcuatus is not considered as a hypospadias subtype in pediatric urology. Therefore, penis arcuatus was not validated nor regarded as a hypospadias subtype in the medical record review. The independent reviews from the two investigators were compared, typing errors were corrected, and disagreements were resolved by consensus decision-making. Subsequently, data were exported for the statistical analyses performed in Stata 13.1.

\section{Statistical analyses}

The accuracy of hypospadias diagnoses and surgical treatment codes was calculated as positive predictive values (PPVs) with 95\% confidence intervals (CIs) overall for diagnosis of hypospadias and surgical treatment of hypospadias, as well as for every specific sub-diagnosis or -surgical treatment code. All PPVs were calculated as the proportion of diagnoses from the DNPR that were confirmed during the medical record review. When assessing the accuracy of the specific subtypes of hypospadias, we used different 
approaches. A total of 440 (88\%) patients were registered with more than one hypospadias diagnosis during the study period, which corresponds well with the clinical practice for diagnosing hypospadias in Denmark. Typically, the patients first have outpatient hospital contact and then, inpatient contact with surgical treatment. Subsequently, there will be at least one - and often many - follow-up outpatient hospital contacts. Therefore, we validated both the first diagnosis and the most severe diagnosis defined as the most proximal location of the external urethral orifice.

We further estimated PPVs stratified by calendar year of first diagnosis (1995-1998, 1999-2003, 2004-2008, and 2009-2012), region of Denmark (North, Central, Southern, Sealand, and Capital), hospital type (university and referral) and department type deciding the location of the external urethral orifice (pediatric urology or pediatric surgery, plastic surgery and general surgery or urology), number of ICD-10 hypospadias diagnoses in the DNPR ( 1 and $\geq 2$ ), response time to our contact (fast if the departments responded after the first contact and slow if they responded after reminders were sent out) and presence of genetic syndromes or other congenital abnormalities.

Finally, in a sensitivity analysis, we assumed a worst-case scenario where all patients with unavailable medical records were classified as not having hypospadias.

\section{Ethical approval}

The study was approved by the Danish Data Protection Agency (Ref. no: 2013-41-1964), the Danish National Board of Health (Ref. no: 3-3013-580/1/), and the State Serum Institute (Ref. no: FSEID-00001380). In accordance with Danish legislation, approval from the Danish Ethics Committee was not required.

\section{Results}

In the study period, 3,700 males were registered with a hypospadias diagnosis and among them 2,956 were registered with a surgical treatment code for hypospadias. We randomly sampled 500 of the 3,700 diagnosed males for this study and among these, 384 were registered with a surgical treatment code for hypospadias.

We successfully retrieved medical records for 463 $(92.6 \%)$ out of the 500 patients from a total of 42 clinical departments in Denmark. Among the 384 patients registered with surgical treatment, we obtained descriptions from surgical treatment of hypospadias on 329 (85.7\%) patients. No departments refused to participate.

Table 1 shows baseline characteristics from the DNPR of the 500 patients stratified by medical record availability. Only for $37(7.4 \%)$ patients, medical records were unavailable, and these were more often diagnosed in the early years of the study period and at referral hospitals.

We confirmed the hypospadias diagnosis for 450 of the 463 patients and found an overall PPV for the hypospadias diagnoses of 97.6\% (95.8\%-98.7\%) (Table 2). Assuming a worst-case scenario where all 37 patients with unavailable medical records were misclassified, the PPV would be $90.0 \%$ (87.0\%-92.3\%).

Figure 1 shows the percentage distribution of the hypospadias subtypes registered in the DNPR (Figure 1A) as well as the subtype of hypospadias according to the medical record review (Figure 1B). We found glandular or coronal hypospadias in $47.2 \%$, corporal hypospadias in $31.8 \%$, penoscrotal hypospadias in 5.0\%, and perineal hypospadias in $0.6 \%$. The PPVs differed according to the specific subtypes; in case of glandular and coronal hypospadias, the PPV was $64.7 \%$ when validating the first hypospadias diagnosis and the PPV for the more severe subtypes ranged from $50.0 \%$ to $72.7 \%$ (Table 2 ).

Table I Medical record availability according to characteristics among 500 patients diagnosed with hypospadias in the Danish National Patient Register, 1995-2012

\begin{tabular}{|c|c|c|}
\hline & \multicolumn{2}{|c|}{ Medical record availability } \\
\hline & $\begin{array}{l}\text { Available } \\
n=463(92.6 \%)\end{array}$ & $\begin{array}{l}\text { Not available } \\
n=37(7.4 \%)\end{array}$ \\
\hline \multicolumn{3}{|l|}{ Year at first diagnosis, $\mathrm{n}(\%)$} \\
\hline $1995-1998$ & $|3|(28.3)$ & $28(75.7)$ \\
\hline 1999-2003 & $122(26.4)$ & $4(10.8)$ \\
\hline 2004-2008 & $125(27.0)$ & $5(13.5)$ \\
\hline $2009-2012$ & $85(18.4)$ & $0(0.0)$ \\
\hline \multicolumn{3}{|l|}{ Region of Denmark, n (\%) } \\
\hline North & $34(7.3)$ & $0(0.0)$ \\
\hline Central & $167(36.1)$ & $8(21.6)$ \\
\hline Southern & $84(18.1)$ & $8(21.6)$ \\
\hline Sealand & $28(6.1)$ & $2(5.4)$ \\
\hline Capital & $150(32.4)$ & $19(51.4)$ \\
\hline \multicolumn{3}{|l|}{ Hospital type, n (\%) } \\
\hline University & $34 \mid(73.7)$ & $13(35.1)$ \\
\hline Referral & $112(26.3)$ & $24(64.9)$ \\
\hline \multicolumn{3}{|l|}{ Department type, n (\%) } \\
\hline Pediatric urology or surgery & $324(70.0)$ & $6(16.2)$ \\
\hline Plastic surgery & $118(25.5)$ & $17(46.0)$ \\
\hline General surgery or urology & $21(4.5)$ & $7(18.9)$ \\
\hline Other & $0(0.0)$ & $7(18.9)$ \\
\hline \multicolumn{3}{|l|}{$\begin{array}{l}\text { Number of ICD-I0 hypospadias } \\
\text { diagnoses in DNPR, } n(\%)\end{array}$} \\
\hline 1 & $53(11.5)$ & $7(18.9)$ \\
\hline$\geq 2$ & $410(88.5)$ & $30(81.1)$ \\
\hline \multicolumn{3}{|l|}{$\begin{array}{l}\text { Genetic syndromes or other } \\
\text { congenital abnormalities, }{ }^{a} \text { (\%) }\end{array}$} \\
\hline Yes & $130(28.1)$ & $6(16.2)$ \\
\hline No & $333(71.9)$ & $31(83.8)$ \\
\hline
\end{tabular}

Note: ${ }^{a}$ Genetic syndromes or congenital abnormalities (Q00-Q99) other than hypospadias (Q54, Q540, Q540A, Q54I, Q542, Q543, Q548, and Q549).

Abbreviation: DNPR, Danish National Patient Register; ICD, International Classification of Diseases. 
Table 2 Positive predictive values of the hypospadias diagnoses among 463 patients in the Danish National Patient Register, 1995-2012

\begin{tabular}{|c|c|c|c|c|c|c|c|c|c|}
\hline & \multirow[t]{2}{*}{ ICD-IO } & \multicolumn{4}{|c|}{ Accuracy of the first diagnosis ${ }^{a}$} & \multicolumn{4}{|c|}{ Accuracy of the most severe diagnosis ${ }^{b}$} \\
\hline & & Sample & Ratioc $^{c}$ & PPV (\%) & $95 \% \mathrm{Cl}(\%)$ & Sample & Ratioc $^{c}$ & PPV (\%) & $95 \% \mathrm{Cl}(\%)$ \\
\hline Overall hypospadias $^{d}$ & Q54 ${ }^{d}$ & 463 & $450 / 463$ & 97.6 & $95.8-98.7$ & 463 & $450 / 463$ & 97.6 & $95.8-98.7$ \\
\hline \multirow[t]{2}{*}{ Glandular or coronal hypospadias } & Q540 & 235 & $152 / 235$ & 64.7 & $58.3-70.6$ & 208 & $150 / 208$ & 72.1 & $65.6-77.8$ \\
\hline & Q540A & & & & & & & & \\
\hline Corporal hypospadias & Q54I & 92 & $50 / 92$ & 54.3 & $43.9-64.4$ & 173 & $98 / 173$ & 56.6 & $49.1-63.9$ \\
\hline Penoscrotal hypospadias & Q542 & 22 & $16 / 22$ & 72.7 & $49.0-88.1$ & 33 & $18 / 33$ & 54.5 & $36.8-71.2$ \\
\hline Perineal hypospadias & Q543 & 2 & $1 / 2$ & 50.0 & - & 8 & $3 / 8$ & 37.5 & 8.7-79.1 \\
\hline \multirow[t]{2}{*}{ Other or unspecified hypospadias ${ }^{\mathrm{e}}$} & Q548 & 112 & $110 / 112$ & 98.2 & $93.1-99.6$ & 41 & $39 / 41$ & 95.1 & $82.2-98.8$ \\
\hline & Q549 & & & & & & & & \\
\hline
\end{tabular}

Notes: ${ }^{2}$ Accuracy of the first ICD-10 diagnosis of hypospadias registered in the DNPR from 1995 to 2012 . ${ }^{b} A c c u r a c y$ of the most severe ICD-I0 diagnosis of hypospadias registered in the DNPR from 1995 to 2012 . 'Ratio denotes confirmed diagnoses/available records. 'Overall PPV for all ICD-I0 codes for hypospadias, Q54, Q540, Q540A, Q54I, Q542, Q543, Q548, and Q549. 'Accuracy of other (Q548) or unspecified hypospadias (Q549) registration in regard to the number of diagnoses that could be confirmed as any form of hypospadias in the medical record review.

Abbreviations: DNPR, Danish National Patient Register; ICD, International Classification of Diseases; PPV, positive predictive value.

A

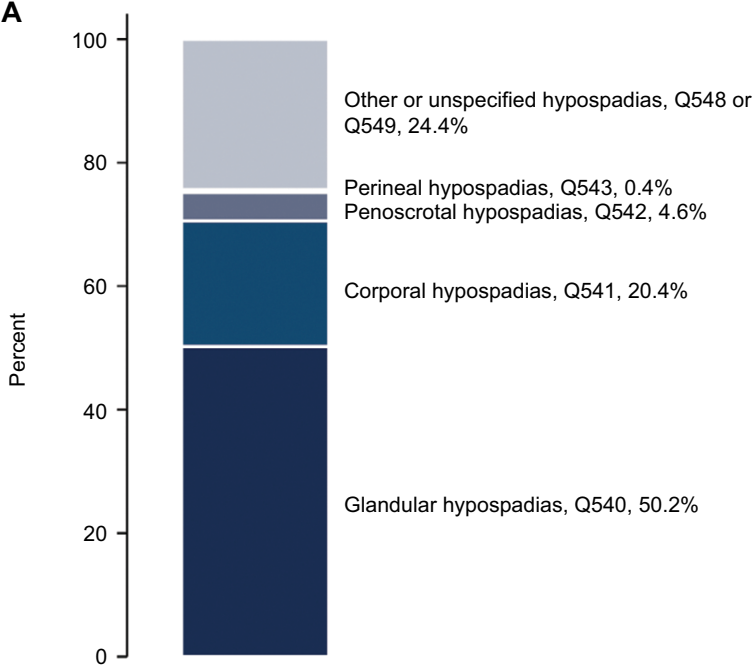

B

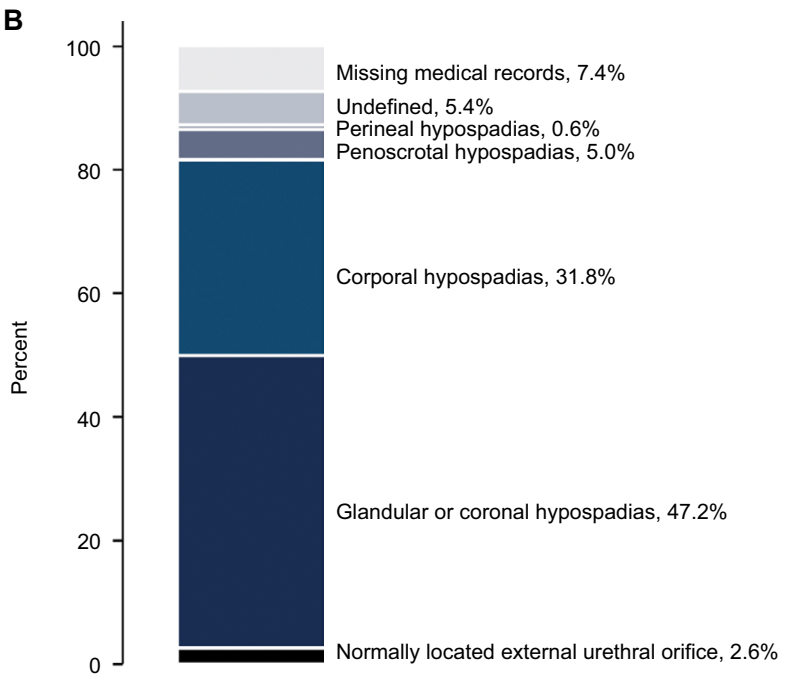

Figure I Percentage distribution of the ICD-10 diagnoses codes for subtypes of hypospadias registered in the Danish National Patient Register (A) and subtype of hypospadias based on medical record review $(\mathbf{B})$.

Abbreviation: ICD, International Classification of Diseases.

A

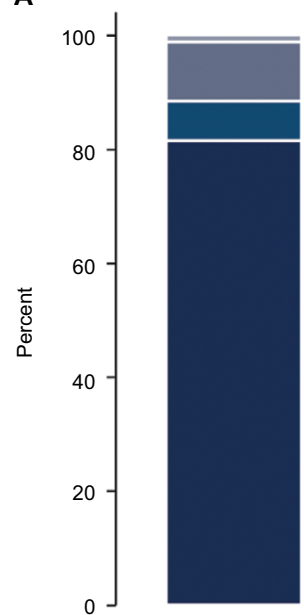

B Surgical treatment, penoscrotal hypospadias, KKGH60C, 1.2\% Surgical treatment, corporal hypospadias, KKGH60B, 10.3\% Surgical treatment, glandular hypospadias, KKGH60A, 7.0\%

Surgical treatment, unspecific hypospadias, KKGH60, 81.5\%

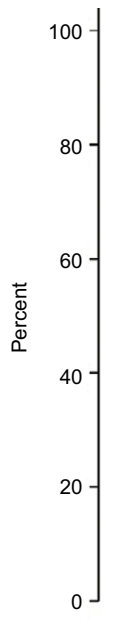

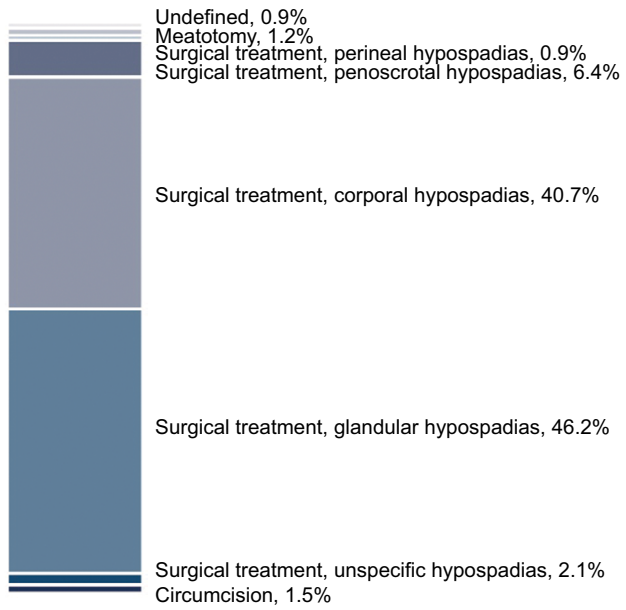

Undefined, $0.9 \%$

Figure 2 Percentage distribution of the operation codes for surgical treatment of hypospadias registered in the Danish National Patient Register (A) and surgical treatment of hypospadias based on medical record review (B). 
Figure 2 presents the percentage distribution of the operation codes for surgical treatment of hypospadias registered in the DNPR (Figure 2A) and the surgical treatment of hypospadias retrieved from the medical record review (Figure 2B). In total, $81.5 \%$ was registered in the DNPR with the unspecific hypospadias operation code KGH60. Table 3 presents the PPVs of surgical treatment of hypospadias. The medical records confirmed 328 of the 329 registered surgical treatments, yielding an overall PPV of 99.7\% (97.9\%-99.9\%). As for the diagnoses, the PPVs of subtypes of surgical treatment were lower than the PPV for overall surgical treatment, decreasing stepwise according to severity (Table 3 ).

Analyses stratified by year at first diagnosis, region of Denmark, hospital type, response time, and presence of genetic syndromes or other congenital abnormalities closely agreed with our main findings (Table 4). However, the PPVs varied according to department type as well as number of hypospadias diagnoses in the DNPR. For general surgery or urology, the PPV was lower than for pediatric surgery and urology and for plastic surgery. For those registered with $\geq 2$ diagnoses, the PPV was 98.0 (96.1\%-99.0\%) compared to $90.6 \%(79.1 \%-96.1 \%)$ for those who had only one diagnosis in the DNPR (Table 4). In total, $28.1 \%$ of the boys were also registered in the DNPR with other congenital abnormalities or genetic syndromes. It is well known that boys born with hypospadias more often have additional congenital abnormalities or that the hypospadias is part of a genetic syndrome. ${ }^{1,2}$ In particular, cryptorchidism and other urogenital anomalies are frequent. ${ }^{3,4}$ Yet, the PPV did not vary between those with isolated hypospadias and those also registered with other congenital abnormalities.

\section{Discussion}

This nationwide, population-based validation study showed that the ICD-10 hypospadias diagnoses and surgical treatments in the DNPR have an overall high PPV.

This study is the first to validate and provide PPVs of hypospadias diagnosis and surgical treatment registrations. A former Danish case-control study by Pedersen et al. indicated a similarly high accuracy. ${ }^{17}$ Their main objective was to examine the risk of hypospadias among boys exposed to antihistamines during pregnancy using data from the DNPR from 1989 to $2003 .{ }^{17}$ As a secondary aim, they investigated the potential misclassification by reviewing medical records for 43 of the 227 hypospadias cases. They did not estimate PPVs, but found that three out of the 43 cases were misclassified.
Later, in a large systematic review of the content in the DNPR, Schmidt et al. recalculated the proportions and found a PPV of $93.0 \%(81.4 \%-97.6 \%){ }^{15}$ However, the study was performed only on data from the North and Central regions in Denmark (i.e., not a random sample of the registry content) and covered an earlier time period.

A major strength of our study was the nationwide population-based design. We were able to study a random sample of all patients recorded with an ICD-10 diagnosis of hypospadias, and successfully retrieved $92.6 \%$ of the requested medical records, thus, limiting the risk of selection bias. Comparison of characteristics among available and unavailable medical records showed that older records were less available and that unavailability was associated with geographical region and type of hospital. In stratified analyses, we found very similar PPVs when we compared PPVs between departments who responded fast versus those who responded slowly to our medical record request. Further, in a rather unlikely worst-case scenario, assuming that all patients with missing information were incorrectly registered, the PPV was still $90.0 \%(87.0 \%-92.3 \%)$. We are therefore quite confident that the presented PPV is not substantially overestimated.

In contrast, the PPVs for subtypes of hypospadias were rather low ranging from $37.5 \%$ to $72.7 \%$, and although the PPVs for surgical treatments were generally higher, they never reached the level of the overall PPVs. However, the sample sizes for penoscrotal and perineal hypospadias were small, rendering uncertain PPVs. Thus, in general, cautious interpretation of research on specific subtypes of hypospadias is warranted. Further, it is important to emphasize that we did not validate the ICD-8 codes, which were used from 1977 to 1994 . Often, register-based studies cover the whole registration period for the DNPR, but our results cannot be extrapolated to the period before 1995 .

Further, it should be noted that we present PPV as the validity measure. The PPVs are influenced by the prevalence of hypospadias in the sample. If one had randomly sampled boys from the entire population, where the prevalence of hypospadias is lower than in this study, the PPVs may also have been lower. However, as this would require a very large sample, such a study seems rather unfeasible. Thus, in this study, we have assessed the validity of the hypospadias diagnoses and surgical treatment codes registered in the DNPR.

Moreover, because of the design, we were not able to study the completeness, e.g., the proportion of patients with hypospadias, who were not registered in the DNPR (false 
Table 3 Positive predictive values of surgical treatment of hypospadias among 329 patients in the Danish National Patient Register, 1995-2012

\begin{tabular}{llllll}
\hline & ICD-I0 & Sample & Ratio $^{\text {a }}$ & PPV (\%) & 95\% Cl (\%) \\
\hline Overall surgical treatment for hypospadias $^{\mathrm{b}}$ & KGH60 & 329 & $328 / 329$ & 99.7 & $97.9-99.9$ \\
Hypospadias, unspecific $^{\mathrm{c}}$ & KGH60 & 268 & $267 / 268$ & 99.6 & $97.4-99.9$ \\
Glandular or coronal hypospadias & KGH60A & 23 & $20 / 23$ & 87.0 & $64.2-96.1$ \\
Corporal hypospadias & KGH60B & 34 & $28 / 34$ & 82.4 & $64.8-92.2$ \\
Penoscrotal hypospadias & KGH60C & 4 & 3 & 75.0 & $4.1-99.5$ \\
Perineal hypospadias & KGH60D & 0 & - & - & - \\
\hline
\end{tabular}

Notes: ${ }^{a}$ Ratio denotes confirmed diagnoses/available records. ' ${ }^{2}$ The overall PPV for all surgical treatment codes (KGH60, KGH60A, KGH60B, KGH60C, and KGH60D) for hypospadias among the 329 patients with available descriptions from surgical treatment. ${ }^{\mathrm{C} T h e} \mathrm{PPV}$ for the unspecific hypospadias operation code KGH60, calculated as the number of boys with a hypospadias operation verified in the medical records/number of boys registered with $\mathrm{KGH} 60$.

Abbreviations: ICD, International Classification of Diseases; PPV, positive predictive value.

Table 4 Positive predictive values of the hypospadias diagnosis stratified by potentially modifying characteristics among 463 patients in the Danish National Patient Register, 1995-2012

\begin{tabular}{|c|c|c|c|}
\hline & \multicolumn{3}{|c|}{ Overall hypospadias diagnoses } \\
\hline & Ratio & PPV (\%) & $95 \% \mathrm{Cl}(\%)$ \\
\hline \multicolumn{4}{|l|}{ Year at first diagnosis } \\
\hline $1995-1998$ & $|27 /| 3 \mid$ & 96.9 & $92.1-98.9$ \\
\hline 1999-2003 & $117 / 122$ & 95.9 & $90.5-98.3$ \\
\hline 2004-2008 & $122 / 125$ & 97.6 & $92.8-99.2$ \\
\hline $2009-2012$ & $84 / 85$ & 98.8 & $92.0-99.8$ \\
\hline \multicolumn{4}{|l|}{ Region of Denmark } \\
\hline North & $33 / 34$ & 97.1 & $81.3-99.6$ \\
\hline Central & $164 / 176$ & 98.2 & $94.5-99.9$ \\
\hline Southern & $81 / 84$ & 96.4 & $89.4-98.9$ \\
\hline Sealand & $28 / 28$ & 100.0 & - \\
\hline Capital & $144 / 150$ & 96.0 & $91.3-98.2$ \\
\hline \multicolumn{4}{|l|}{ Hospital type } \\
\hline University & $336 / 341$ & 98.5 & $96.5-99.4$ \\
\hline Referral & $114 / 122$ & 93.4 & $87.4-96.7$ \\
\hline \multicolumn{4}{|l|}{ Department type } \\
\hline Pediatric urology or surgery & $320 / 324$ & 98.8 & $96.7-99.5$ \\
\hline Plastic surgery & $116 / 118$ & 98.3 & $93.4-99.6$ \\
\hline General surgery or urology & $|4 / 2|$ & 66.7 & 44. I-83.6 \\
\hline \multicolumn{4}{|c|}{ Number of ICD-I0 hypospadias diagnoses in DNPR } \\
\hline I & $48 / 53$ & 90.6 & $79.1-96.1$ \\
\hline$\geq 2$ & $402 / 410$ & 98.0 & $96.1-99.0$ \\
\hline \multicolumn{4}{|l|}{ Response time } \\
\hline Fast & $372 / 382$ & 97.4 & $95.2-98.6$ \\
\hline Slow & $78 / 81$ & 96.4 & 89.0-98.8 \\
\hline \multicolumn{4}{|c|}{ Genetic syndromes or other congenital abnormalities ${ }^{b}$} \\
\hline Yes & $126 / 130$ & 96.9 & $92.0-98.8$ \\
\hline No & $224 / 333$ & 97.3 & $94.9-98.6$ \\
\hline
\end{tabular}

Notes: a Overall hypospadias diagnoses, any type; Q54, Q540, Q540A, Q54I,

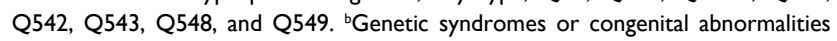
(Q00-Q99) other than hypospadias (Q54, Q540, Q540A, Q54I, Q542, Q543, Q548, and Q549).

Abbreviations: DNPR, Danish National Patient Register; ICD, International Classification of Diseases; PPV, positive predictive value.

negative cases), as the study population was defined as patients registered with ICD-10 codes in the DNPR. This needs to be considered when using the ICD-10 codes for hypospadias ascertainment in register-based research.

\section{Conclusion}

We found a very high overall PPV of hypospadias diagnosis and surgical treatment registrations. Thus, the DNPR is a valid tool for observational research on hypospadias. However, in future studies on specific subtypes of hypospadias, there should be awareness of the low PPVs.

\section{Disclosure}

The authors report no conflicts of interest in this work.

\section{References}

1. Pedersen CB, Gotzsche H, Moller JO, Mortensen PB. The Danish Civil Registration System. A cohort of eight million persons. Dan Med Bull. 2006;53(4):441-449.

2. Pedersen CB. The Danish Civil Registration System. Scand J Public Health. 2011;39(7 Suppl):22-25.

3. Lynge E, Sandegaard JL, Rebolj M. The Danish National Patient Register. Scand J Public Health. 2011;39(7 Suppl):30-33.

4. Andersen TF, Madsen M, Jorgensen J, Mellemkjoer L, Olsen JH. The Danish National Hospital Register. A valuable source of data for modern health sciences. Dan Med Bull. 1999;46(3):263-268.

5. Lund L, Engebjerg MC, Pedersen L, Ehrenstein V, Norgaard M, Sorensen HT. Prevalence of hypospadias in Danish boys: a longitudinal study, 1977-2005. Eur Urol. 2009;55(5):1022-1026.

6. Kraft KH, Shukla AR, Canning DA. Hypospadias. Urol Clin North Am. 2010;37(2):167-181.

7. van der Zanden LF, van Rooij IA, Feitz WF, Franke B, Knoers NV, Roeleveld N. Aetiology of hypospadias: a systematic review of genes and environment. Hum Reprod Update. 2012;18(3):260-283.

8. Jensen MS, Wilcox AJ, Olsen J, et al. Cryptorchidism and hypospadias in a cohort of 934,538 Danish boys: the role of birth weight, gestational age, body dimensions, and fetal growth. Am J Epidemiol. 2012;175(9):917-925.

9. Arendt LH, Ramlau-Hansen CH, Wilcox AJ, Henriksen TB, Olsen J, Lindhard MS. Placental weight and male genital anomalies: a nationwide Danish cohort study. Am J Epidemiol. 2016;183(12):1122-1128.

10. Schnack TH, Zdravkovic S, Myrup C, et al. Familial aggregation of hypospadias: a cohort study. Am J Epidemiol. 2008;167(3): 251-256.

11. Schnack TH, Poulsen G, Myrup C, Wohlfahrt J, Melbye M. Familial coaggregation of cryptorchidism and hypospadias. Epidemiology. 2010;21(1):109-113.

12. Sorensen HT, Pedersen L, Norgaard M, Wogelius P, Rothman KJ. Maternal asthma, preeclampsia and risk of hypospadias. Epidemiology. 2005;16(6):806-807. 
13. Weidner IS, Moller H, Jensen TK, Skakkebaek NE. Risk factors for cryptorchidism and hypospadias. J Urol. 1999;161(5):1606-1609.

14. Morales-Suarez-Varela MM, Toft GV, Jensen MS, et al. Parental occupational exposure to endocrine disrupting chemicals and male genital malformations: a study in the Danish National Birth Cohort study. Environ Health. 2011;10(1):3.

15. Schmidt M, Schmidt SA, Sandegaard JL, Ehrenstein V, Pedersen L, Sorensen HT. The Danish National Patient Registry: a review of content, data quality, and research potential. Clin Epidemiol. 2015;7:449-490.
16. Harris PA, Taylor R, Thielke R, Payne J, Gonzalez N, Conde JG. Research electronic data capture (REDCap)--a metadata-driven methodology and workflow process for providing translational research informatics support. J Biomed Inform. 2009;42(2):377-381.

17. Pedersen L, Norgaard M, Skriver MV, Olsen J, Sorensen HT. Prenatal exposure to loratadine in children with hypospadias: a nested casecontrol study within the Danish National Birth Cohort. Am J Ther 2006;13(4):320-324.
Clinical Epidemiology

\section{Publish your work in this journal}

Clinical Epidemiology is an international, peer-reviewed, open access, online journal focusing on disease and drug epidemiology, identification of risk factors and screening procedures to develop optimal preventative initiatives and programs. Specific topics include: diagnosis, prognosis, treatment, screening, prevention, risk factor modification,

Submit your manuscript here: https://www.dovepress.com/clinical-epidemiology-journal

\section{Dovepress}

systematic reviews, risk and safety of medical interventions, epidemiology and biostatistical methods, and evaluation of guidelines, translational medicine, health policies and economic evaluations. The manuscript management system is completely online and includes a very quick and fair peer-review system, which is all easy to use. 